\title{
Jorge León Arguedas (9 diciembre 1916 - 5 junio 2013) ${ }^{1}$
}

\author{
«Erudito, científico riguroso, metódico, mesurado y \\ juicioso en sus opiniones, humilde hasta los tuétanos
} y generoso en compartir sus conocimientos, como un auténtico maestro»

Hilje (2013)

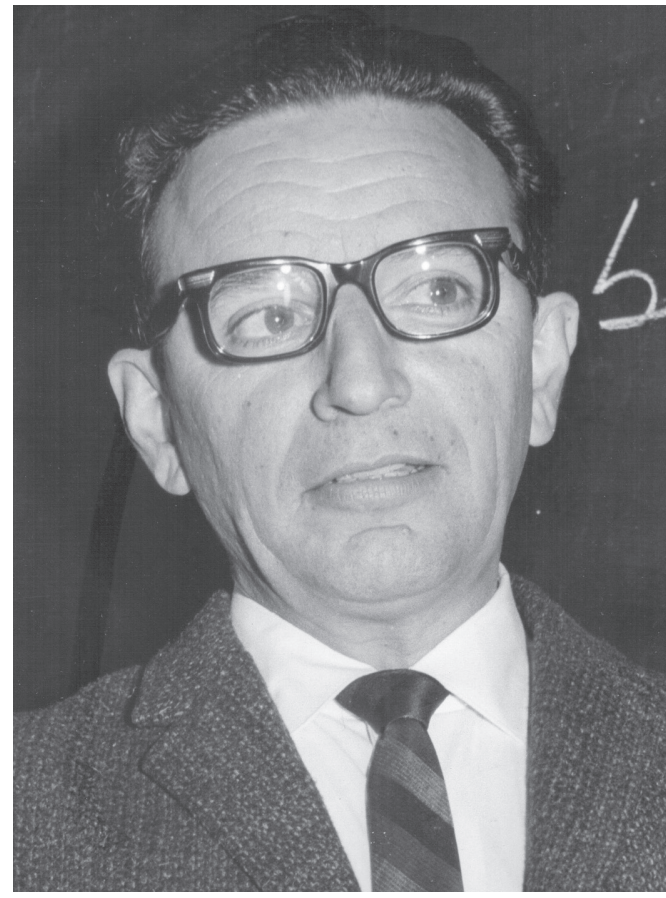

Dr. Jorge León, 1970. Foto cortesía Familia León Saénz.

El Dr. Jorge León, como se le conoció en el mundo científico, nació cerca de las 5 a.m., en el centro de la aldeana, pintoresca, colonial y aún villa de Barva de Heredia, Costa Rica, el 9 de diciembre de 1916 y fue bautizado con el largo nombre, como era usual en esa época, de Jorge José María de Jesús León Arguedas. Fueron sus progenitores don Tomás León y doña Josefa Arguedas. Con 29 años recién cumplidos, se unió en feliz matrimonio en la ciudad de Heredia el 24 de enero de 1946 con María Eugenia del Socorro Sáenz Ulloa, con quien procreó tres hijos: Jorge Eduardo (1946), Ana Teresa (1954) y Roberto (1955).

Don Jorge asistió a la Escuela Pedro Murillo Pérez de Barva, para posteriormente ingresar a la Escuela Normal de Heredia, donde se graduó como maestro en diciembre de 1936, al igual que lo haría su futura esposa años más tarde. Su primer oficio como educador lo ejerció a partir del año siguiente en la escuela primaria de Juan Viñas (Jiménez de Cartago), bautizada durante su estancia, en 1939, con el nombre de Escuela Cecilio Lindo Morales.

Estando en esta localidad, la escuela recibió una nota de parte de don Juvenal Valerio Rodríguez, entonces Director del Museo Nacional, quien solicitaba el envío de un ejemplar vegetal que se encontraba en esta localidad. Don Jorge atendió esta solicitud y en uno de sus viajes a San José se la entregó personalmente. A partir de ese entonces nació

1. Dedicado, con estima y aprecio, a don Gilbert Fuentes G., Carlos Ossenbach S. y Julián Monge-Nájera, por su disposición permanente y generosa para compartir sus conocimientos y documentos con quien los solicite. 
una amistad entrañable entre ambos, al punto de que don Jorge llegó a considerar a don Juvenal como su principal mentor. Posteriormente don Juvenal le ofreció trabajar en el Herbario Nacional, donde don Jorge laboró como botánico entre 1940 y 1943, encargado de exploración (con poco más de 4000 especímenes de plantas recolectados) y de las labores curatoriales usuales en un herbario. Durante este tiempo también ejerció como docente en el Liceo Nocturno Domingo Faustino Sarmiento en San José, fue consultor de la Secretaría de Agricultura y realizó misiones de estudio de carácter botánico, tanto en nuestro país como en El Salvador y Guatemala. En mayo de 1942, y con apenas 25 años de edad, terminó de escribir su primer libro Nueva Geografia de Costa Rica, en el cual tuvo la oportunidad de aplicar los conocimientos adquiridos en sus diversos viajes a lo interno del país como explorador botánico del Museo Nacional. Esta publicación salió de los talleres de impresión al año siguiente y fue utilizada con mucho éxito en los colegios del país, llegando a editarse en diez ocasiones (hasta 1952).

Desde el inicio don Juvenal Valerio supo aquilatar la personalidad y la calidad del trabajo de don Jorge León, así como vislumbrar su brillante futuro, cuando se refirió a él, con motivo de su ingreso como Jefe de la Sección Botánica del Museo Nacional, en sustitución del Dr. Alexander Skutch, con las siguientes palabras:

“(...), vino a trabajar con nosotros, como Jefe de la Sección Botánica, don Jorge León Arguedas, elemento nuevo, verdadero autodidacto que después de descollar en sus tareas docentes y hecho a las disciplinas de la Historia Natural, ha encontrado en el medio que le proporciona el Museo su mejor apoyo para desarrollar las excelentes capacidades de investigador que lo adornan.

Viéndolo trabajar no podemos, quienes ya pertenecemos a la vieja generación, más que sentirnos verdaderamente satisfechos de pensar que las investigaciones botánicas no han de decaer si por siempre pueden descansar en jóvenes [23 años] como León Arguedas." (Valerio, 1942a).
En un informe posterior, don Juvenal va más allá y llega a afirmar con absoluta certeza que don Jorge León puede llamarse: “el mejor botánico nacional de la tercera generación (...)." (Valerio, 1942b).

En 1943 fue nombrado por el Secretario de Agricultura para formar parte de una comisión especial de notables, que debería valorar aspectos relacionados con la conservación y el estudio de los excepcionales bosques de robles existentes en el trayecto en construcción de la Carretera Panamericana (Alfaro et al., 1944). Los otros miembros de esta comisión fueron, nada menos que Clodomiro Picado, Anastasio Alfaro, Carlos Lankester, Otón Jiménez y Rafael A. Chavarría.

Entre 1943 y 1947 laboró como asistente en el Programa de Planeamiento del Instituto de Asuntos Interamericanos con sede en Costa Rica, donde estuvo a cargo del establecimiento de ensayos y la introducción de nuevas variedades vegetales, así como de la supervisión de trabajos de campo y la realización de estudios regionales de desarrollo agrícola. En esta época (1945-1946) tuvo la oportunidad de recibir un adiestramiento especial por parte del Departamento de Agricultura de los EE.UU. en la ciudad de Washington, D.C.

En los siguientes cinco años (1947-1951) don Jorge trabajó en Turrialba en el Instituto Interamericano de Ciencias Agrícolas (IICA, hoy CATIE) como encargado de la introducción, el establecimiento y el mantenimiento de plantas, así como de las actividades relacionadas con intercambios de semillas. Aquí creó y consolidó tanto el jardín botánico como el banco de germoplasma de recursos fitogenéticos de esta institución, cuya importancia y relevancia -pasada, actual y futura- a nivel internacional se destacan en las publicaciones de Ebert (2008) y Ebert et al. (2007).

Posteriormente viajó a EE.UU. a realizar estudios académicos en la Universidad de Washington, en estrecha relación con el Jardín Botánico de Missouri en la ciudad de San Luis, con una beca de la Fundación John Simon Guggenheim. Don Jorge culminó sus estudios de doctorado con la guía del Dr. Robert E. 
Woodson Jr. y del Dr. Edgar Anderson, en la Escuela de Graduados de Artes y Ciencias, con la presentación y defensa de su trabajo de tesis The genus Inga Scop. in Central America and The West Indies, la tarde del 19 de mayo de 1953, en el aula 308 del edificio Rebstock Hall. De este trabajo llegó a publicarse más tarde un extenso artículo (León, 1966). Don Otón Jiménez Luthmer (1895-1988), reconocido botánico costarricense y miembro de Silla de la Academia de Geografía e Historia de Costa Rica, emitió el siguiente criterio con respecto a la calidad de este trabajo:

"Su tesis para doctorarse (...), por su laboriosidad y erudición, coloca a nuestro compatriota a la par de los más eminentes especialistas en este ramo del mundo entero, lo que es gloria no sólo de nuestra pequeña Costa Rica, sino del Mundo Hispano.” (Jiménez, 1967).

A su regreso a Costa Rica se reincorporó a su trabajo en el IICA, donde, entre otras labores, tuvo a su cargo -entre 1953 y 1955 - el establecimiento y mantenimiento de colecciones vegetales, especialmente de variedades de café. De 1955 a 1962 asumió el cargo de Director del Departamento de Fitotecnia de esta organización, colaborando a su vez en la elaboración del currículum de la naciente Escuela para Graduados, en la cual fue profesor del curso Método Científico. Durante estos años fue profesor tutor de tesis, entre otros, del entonces joven estudiante Luis A. Fournier O. (1935-2002), de grata memoria en la Escuela de Biología de la Universidad de Costa Rica (UCR) y sobre el que presentó en público y escribió una semblanza (León, 2006). Adicionalmente, fue miembro del Comité Internacional para la Nomenclatura de Plantas Cultivadas (desde 1958).

Entre 1962 y 1968 trasladó su residencia a Lima (Perú), para ejercer el puesto de Director del Programa Regional de Investigaciones del IICA (Zona Andina). Allí tuvo a cargo la promoción e investigación agrícola en la región (Bolivia, Colombia, Ecuador, Perú y Venezuela) por medio de la organización de cursos,

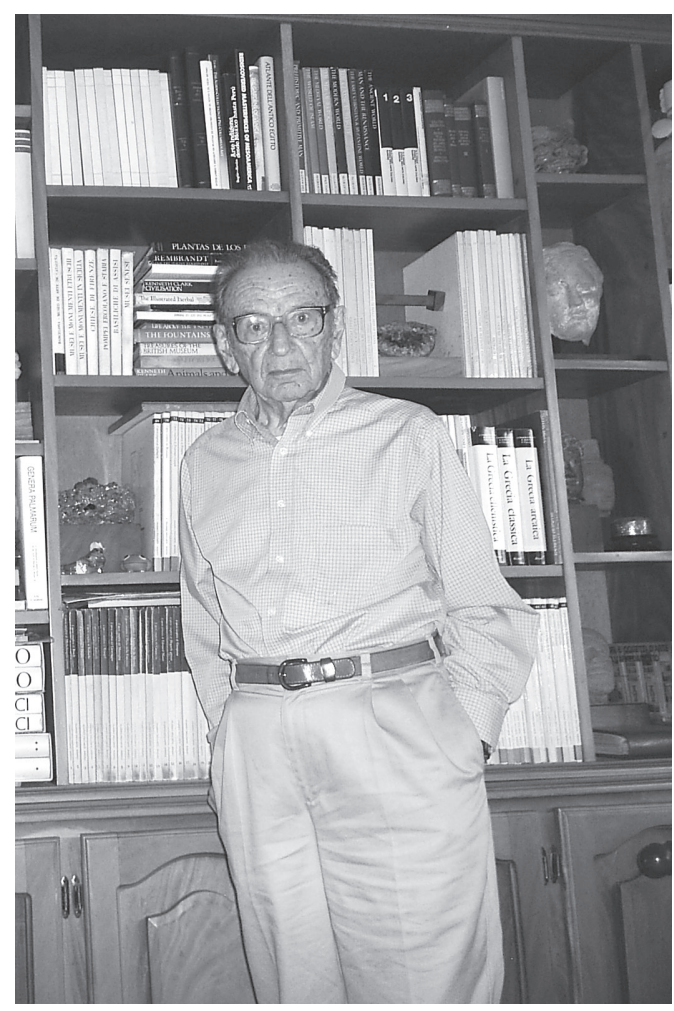

Dr. Jorge León, 2007. Foto cortesía Familia León Saénz.

reuniones, publicaciones y otorgamiento de becas. Adicionalmente asumió la dirección de investigación en plantas andinas nativas, incluyendo el establecimiento de colecciones de germoplasma, así como la realización de estudios agronómicos y de selección. Como se reconoce en el prólogo de la publicación de Tapia (2000):

"El Dr. Jorge León, costarricense, secundado por el Ing. Julio Rea, boliviano, fueron los pioneros en la gestión interandina para recuperar estas especies. El Programa de Cultivos Andinos del IICA no sólo recolectó el material de estas especies, sino que capacitó e inspiró a muchos jóvenes agrónomos de la década del sesenta."

Por esa época don Otón Jiménez escribió:

"La labor de Jorge León, en cambio, es tan valiosa como desconocida de sus compatriotas, 
debido a la sencillez y humildad de su carácter, como es el de los sabios de verdad. Al presente trabaja en Lima, la Zona Andina del Instituto Interamericano de Ciencias Agrícolas. Desde esta posición ha contribuido con maravillosos estudios sobre las Plantas Alimenticias, y otras, de esa interesante región.” (Jiménez, 1967).

De Lima se trasladó a Roma (Italia) a trabajar para la Organización Mundial para la Agricultura y la Alimentación (FAO) los siguientes ocho años (1968-1976). Allí ocupó los puestos de Jefe del Programa de Introducción de Plantas, Director de la Unidad de Ecología de Plantas y Recursos Genéticos, y Jefe de la Oficina de Intercambio de Germoplasma. Sus labores en esta organización le llevaron a participar en múltiples trabajos en todo el mundo, que involucraron el planeamiento y la ejecución de programas ligados a las temáticas de introducción, ecología, colección, conservación e inventarios de plantas, supervisión de intercambios de semillas, diversificación de cultivos y exploración de recursos fitogenéticos.

En 1976 regresó a Costa Rica para trabajar en el Centro Agronómico Tropical de Investigación y Enseñanza (CATIE) en calidad de Jefe de la Unidad de Recursos Genéticos. Aquí tuvo a su cargo el establecimiento de proyectos de colecciones de plantas, laboratorios y cuartos de semillas, junto a labores de enseñanza y tutoría a estudiantes de posgrado de esta institución. En 1979 ejerció el puesto de Presidente del Comité Internacional de Germoplasma de Café. En 1982 ocupó la jefatura del Departamento de Desarrollo de Recursos para la Investigación y la Docencia, y en 1983 se acogió a la jubilación. Desde entonces colaboró ad honorem con quienes se lo solicitaron en aspectos de docencia, investigación, gestión y fortalecimiento institucional, sin dejar de continuar elaborando artículos y libros en sus áreas de interés.

Don Jorge describió y publicó los siguientes 17 táxones nuevos (todos con el nombre "J. León" al final, como autoridad científica): En el trabajo de tesis (artículo de 1966), las especies Inga allenii, I. mortoniana, I. squamigera e I. tenuipedunculata, las subespecies Inga vera ssp. eriocarpa e I. vera ssp. spuria y las siguientes siete series de la Sección Inga del género Inga (Fabaceae): Ser. 1. Punctatae, Ser. 2. Multijugae, Ser. 3. Densiflorae, Ser. 5. Acuminatae, Ser. 6. Pilosulae, Ser. 8. Goldmanianae y Ser. 10. Spectabiles; también una combinación nueva: Pithecellobium gigantifoliolum (Schery) J. León, a partir del nombre Inga gigantifoliola Schery. En otras fuentes describió Alfaroa manningii J. León (Juglandaceae), Guarea turrialbana J. León (Meliaceae) y Theobroma mammosum Cuatrec. \& J. León (Sterculiaceae) (León, 1949, 1953, 1960, 1966).

Su actividad como recolector de especímenes vegetales, especialmente en las décadas de 1940 y 1950 , es visible en varios herbarios del continente; por ejemplo: Herbario Nacional de Costa Rica (CR, ca. 2400 especímenes, 19381994), Herbario Paul C. Standley de la Escuela Agrícola Panamericana en Honduras (EAP, ca. 1400 ejemplares, 1941-1968), Herbario Luis A. Fournier de la Universidad de Costa Rica (USJ, ca. 780 especímenes, 1948-1961) y Herbario del Jardín Botánico de Missouri en San Luis, EE.UU. (MO, ca. 158 especímenes, 1939-1989).

La vocación docente lo acompañó a lo largo de su vida, dado que gustaba de compartir sus conocimientos por medio de charlas y cursos. Entre 1956 y 1961 fue profesor de los cursos Cultivos Tropicales y Métodos de Investigación en la Escuela para Graduados del IICA. Durante su estancia en Perú fue profesor del curso Métodos de Investigación (1962 a 1968) en la Universidad Nacional Agraria La Molina (Perú). Entre 1978 y 1982 impartió el curso Recursos Genéticos, en el Programa Conjunto de Estudios de Posgrado en Ciencias Agrícolas y Recursos Naturales de la UCR y el CATIE. En la UCR fue docente ad honorem en las cátedras de Conservación de Recursos Fitogenéticos (Escuela de Fitotecnia, 1982) y Genética (Escuela de Biología, 1983-1988). En la Escuela de Biología colaboró, adicionalmente, tanto a nivel de grado como de posgrado, en las investigaciones relacionadas con los 
recursos genéticos, especialmente pejibaye. Entre las charlas y conferencias magistrales que don Jorge dictó en esta última institución académica en sus últimos años están las siguientes: Semblanza sobre Luis Fournier Origgi (2005), Origen de la agricultura con énfasis en Mesoamérica (2006), y Recursos fitogenéticos: orígenes, extensión y futuro de su uso y conservación (2009).

Gran parte del legado de don Jorge León quedó plasmado en múltiples publicaciones. Entre éstas se destacan (al menos una por década) las siguientes: La educación en Costa Rica (1937), Nueva geografia de Costa Rica (1943), Land utilization in Costa Rica (1948), Plantas alimenticias andinas (1964), Fundamentos botánicos de los cultivos tropicales (1968, versiones revisadas y aumentadas publicadas con el título Botánica de los cultivos tropicales en 1987 y 2000), Los recursos genéticos de las plantas cultivadas de América Central (1979, en colaboración con J. Engels y H. Goldbach), Lost crops of the Incas (1989, con H. Popenoe, S.R. King, L.S. Kalinowski y N.D. Vietmeyer), Aportes de Mesoamérica a la agricultura mundial (1998), Los nombres comunes de las plantas en Costa Rica (2000, con L.J. Poveda Álvarez), La exploración botánica de Costa Rica en el siglo XIX (2002, capítulo de la obra "Ciencia y técnica en la Costa Rica del siglo XIX”, editado por G. Peraldo), y Las plantas más atractivas del Parque Nacional Volcán Poás (2008, con L.J. Poveda Álvarez y P. Sánchez-Vindas).

De gran importancia e interés fueron también sus trabajos de traducción. Entre éstos se destacan los siguientes títulos: Código Internacional de Nomenclatura de las Plantas Cultivadas (1962), Viajes de Cockburn y Lièvre por Costa Rica (1962, con su esposa Maruja Sáenz), Las plantas cultivadas de México, Guatemala y Colombia (1964, de M. Bukasov), Nuevos conceptos sobre la taxonomía de las plantas superiores (1967, de J. Heslop-Harrison), Isla del Coco, relato de una visita hecha en 1836 por el Dr. John Coulter (1996), y La América Central (2011, de A.S. Oersted). Adicionalmente, gestionó ante el Sr. José Dávila
Solera la traducción, del alemán al español, del artículo de Carl Hoffmann Una excursión al volcán Barva de Costa Rica en 1855 (León, 1942).

Sus habilidades como editor quedaron plasmadas en las siguientes obras de la FAO: Handbook of plant introduction in tropical crops (1974), Guidelines for seed exchange and plant introduction in tropical crops (1986, con L.A. Withers) y Cultivos marginados. Otra perspectiva de 1492 (1992, con J.E. Hernández Bermejo). También perteneció al consejo editorial de las revistas Turrialba, Economic Botany, Geografía Agrícola (México) y Revista de Biología Tropical (1993-2013). Además, fungió como editor de las publicaciones del IICA Café-Coffee y Tropical Root and Tuber Crops Newsletter. También realizó consultorías para la Academia Nacional de Ciencias (NAS) de los EE.UU. (en cultivos andinos) y la Agencia Internacional para el Desarrollo (AID) de ese mismo país (sobre recursos genéticos en Perú).

Don Jorge fue miembro, entre otras, de las siguientes organizaciones: Society for Economic Botany, Academia de Ciencias de Nueva York, Sociedad Linneana de Londres (Fellow of the Linnean Society), Comisión Nacional de Recursos Fitogenéticos de Costa Rica (miembro fundador y primer presidente), Fundación para el Fomento y Promoción de la Investigación y Transferencia de la Tecnología Agropecuaria (Fittacori) e Instituto Nacional de Biodiversidad (INBio, miembro fundador $\mathrm{y}$ vicepresidente de la junta directiva).

Sus valiosos aportes a la humanidad en diversos campos, tanto de las ciencias naturales como sociales (botánica, agricultura, promoción y conservación de recursos fitogéneticos locales, historia, geografía y etnobotánica) fueron dichosamente reconocidos en vida, especialmente en las últimas dos décadas, cuando recibió, entre otras, distinciones y reconocimientos de las siguientes organizaciones: Ministerio de Agricultura y Ganadería (1982), Sociedad Linneana de Londres (1983, Fellow of the Linnean Society), Geógrafos de Costa Rica (1984), entes patrocinadores del First International Field Study of Tropical Fruits 
and Plants realizado en Costa Rica (1984), Escuela Agrícola Panamericana El Zamorano (1992, Wilson Popenoe Award), CATIE (1993, 1999 y 2001), Colegio de Ingenieros Agrónomos de Costa Rica (1994, Miembro Honorario), The Society for Economic Botany (1994, President's Award), Academia Nacional de Ciencias (1998, Miembro Honorario), Instituto Costarricense de Cultura Hispánica (1999, Costarricense Distinguido), Fittacori (1999), Instituto Nacional de Biodiversidad (1999), Escuela Centroamericana de Geología de la UCR (2003), Ministerio de Ciencia y Tecnología (2006), IICA (2006, Medalla Agrícola Interamericana), Escuela de Biología de la UCR (2006 y 2009), IICA y UNA (2007, Medalla Agrícola Nacional), Centro de Investigaciones Geofísicas de la UCR (2007), Oficina Nacional de Semillas (2008) y patrocinadores de las Jornadas de Tradición Alimentaria: Naturaleza, creatividad e identidad (2009), y Revista de Biología Tropical (2010). En su honor fueron nombradas dos especies de árboles: Inga leonis N. Zamora (Fabaceae) y Licaria leonis Gómez-Laur. \& A. Estrada (Lauraceae); así como una especie de insectos: Meniscomorpha leoni Ugalde \& Gauld. Asimismo, el Jardín Botánico del CATIE lleva el nombre del Dr. Jorge León (Zamora, 1990; Gómez-Laurito y Estrada, 2002; Ugalde y Gauld, 2002; Ebert et al., 2007).

Otro aspecto interesante de destacar de la obra del Dr. León es la biblioteca personal, que inició hacia 1935 siendo aún estudiante de la Escuela Normal y luego como funcionario del Museo Nacional. En ese entonces su colección se concentró en publicaciones de historia y geografía de Costa Rica, que abarca el período de 1830 a 1950 . A partir de 1940 incluyó la botánica como otra de sus áreas de interés, abarcando temas de conservación de recursos fitogenéticos, sistemática, fisiología, descripción de la flora, etnobotánica con énfasis en América Latina, agricultura (principalmente de café y cacao), arqueología de los pueblos indoamericanos, arte y arquitectura europea. La colección incluye también algunos libros del siglo XVIII y XIX, una colección importante de más de dos centenares de mapas, unas 300 fotografías de Costa Rica en la década de 1940, datos meteorológicos históricos, documentos con estadísticas de Costa Rica, dibujos (varios de su autoría) y correspondencia. En total, se estima que esta valiosa colección abarca unos 12000 ítems (LeoLibri, 2013).

Sin duda, como refiere el Dr. Luko Hilje (2013) en un emotivo artículo sobre don Jorge:

"(...), su lucidez y enciclopedismo lo con-
virtieron por mucho tiempo en un referente
imprescindible para entender numerosos aspec-
tos de nuestra historia natural, así como de los
aportes de varios naturalistas, tanto extranje-
ros como nacionales, en el conocimiento de
nuestra biodiversidad."

Como persona, don Jorge se caracterizó siempre por su gran personalidad, caballerosidad, humildad y, sobre todo, por su generosidad y calidad humana, sin olvidar mencionar su fino y sano sentido del humor. $\mathrm{Al}$ respecto no encuentro palabras más ceñidas a la realidad que las que mencionó el M.Sc. Marco Chaves Solera en 2006, con ocasión del homenaje que se le hizo a don Jorge por parte del Ministerio de Ciencia y Tecnología:

"Digno de resaltar además de lo anotado en materia técnica respecto al Dr. León, es su singular personalidad, caballerosidad y el gran sentido humano que lo caracterizan, ingredientes todos que tipifican a una persona de enorme estatura moral y capacidad intelectual, dimensiones propias apenas de los seres excepcionales.

En la persona del Dr. León Arguedas como en otros personajes de nuestro país, no muchos por cierto, se materializa aquello de que los grandes hombres no son los que más se empeñaron y esforzaron por hacerse sentir, ni tampoco los que ostentaron muchos títulos, condecoraciones y posiciones, sino más bien aquellos que como el Dr. León, ofrecieron con humildad y capacidad su esfuerzo y contribución al progreso de la patria y de los pueblos, poseyendo el 'talento del silencio'. (...)". 
Los que deseamos recordar su imagen y su voz, así como las personas que no tuvieron la oportunidad de conocerlo personalmente, podemos ver la semblanza que le hizo el programa televisivo Espectro de Canal 15 de la UCR (http://goo.gl/kp8EB), así como su participación en los vídeo-documentales de la UNED "Historia de un desafío" (http:/goo. gl/306FZ) y "Defendiendo gigantes" (http:// goo.gl/JCPzp).

Finalmente sólo me resta decir ¡Muchas gracias! don Jorge, por sus valiosos aportes y contribuciones a Costa Rica y al mundo, y muy especialmente por sus múltiples enseñanzas y su amistad sincera para con todos aquellos que tuvimos la dicha de conocerlo y tratarlo personalmente.

"La obra de don Jorge León no acabó, está aún viva, pues plantó su semilla en tierra fértil: sus estudiantes, compañeros y discípulos, y como bien lo expresara Freeman: 'Las semillas son siempre fuerzas positivas y creadoras. Ellas son el germen de la vida, el principio y el fin, el fruto de la cosecha de ayer y la promesa del mañana.' "' Chaves (2006).

\section{AGRADECIMIENTOS}

A doña Maruja Sáenz Ulloa y don Jorge León Sáenz, por su valiosa colaboración en el suministro de gran parte de la información que aquí se menciona, así como por las valiosas observaciones y recomendaciones que le hicieron a la versión preliminar del texto de esta memoria. De igual manera a Alfredo Cascante y George E. Pilz por el envío de la información solicitada sobre los especímenes botánicos recolectados por don Jorge León, que se hallan en los herbarios CR, EAP y USJ. Por último, y no por ello menos importante, deseo también agradecer al equipo de trabajo de la Unidad de Referencia y Documentación del Sistema de Bibliotecas, Documentación e Información de la UCR por la eficiente labor de localización de varios de los documentos aquí referenciados.

\section{REFERENCIAS}

Alfaro, A., Lankester, C. H., Chavarría, R. A., Jiménez, O., \& León, J. (1944). Informe sobre los robledales de Copey. Revista del Instituto de Defensa del Café de Costa Rica, XIV, 114(mayo), 293-296.

Chaves, S. M. (2006). Semblanza [del] Dr. Jorge León Arguedas. Homenaje en ocasión del Día Nacional de la Ciencia y la Tecnología. Ministerio de Ciencia y Tecnología (MICIT). Recuperado de: http://goo.gl/muv01

Ebert, A. W. (2008). Flujos de germoplasma facilitado por el Centro Agronómico de Investigación y Enseñanza dentro y fuera de Latinoamérica (Informe Especial Recursos Fitogenéticos. Recursos Naturales y Ambiente, n. ${ }^{\circ}$ 53, 62-71). Turrialba: CATIE. Recuperado de: http://goo.gl/7GZ73

Ebert, A. W., Astorga, C., Ebert, I. C. M., Mora, A., \& Umaña, C. (2007). Securing our future. CATIE's germplasm collections / Asegurando nuestro futuro. Colecciones de germoplasma del CATIE. (Serie Técnica. Boletín Técnico, n. $\left.{ }^{\circ} 26\right)$. Turrialba: CATIE. 204 pp. Recuperado de: http://goo.gl/tDx9T

Gómez-Laurito, J., \& Estrada, J. (2002). Licaria leonis (Lauraceae), una nueva especie del Pacífico costarricense y algunas notas sobre Licaria multinervis H. Kurz. Lankesteriana, 3, 5-9. Recuperado de: http://goo.gl/yujac

Hilje, Q., L. (2013, 11 de junio). Ha partido un sabio. Diario digital ElPaís.cr. Recuperado de: http://goo.gl/qXUpv

Jiménez, O. (15 de agosto de 1967). Al hablar del Herbario Nacional hay que hacer mención de nombres de distinguidos botánicos. Periódico La Nación (Costa Rica), p. 17. Recuperado de: http://goo.gl/YhNsU5

LeoLibri (2013). Biblioteca Jorge León Arguedas. Recuperado de: http://leolibri.net/origen.php

León, J. (1942). Sección Botánica. In R. J. Valerio (1942). Informe rendido a la Secretaría de Educación Pública sobre la labor realizada en 1940. San José Costa Rica: Museo Nacional. Serie de Historia del Museo, I(III), 6 .

León, J. (1949). Una especie nueva de Theobroma. (Serie Boletín Técnico, $\left.n .^{\circ} 2\right)$. Turrialba: Instituto Interamericano de Ciencias Agrícolas. 6 pp.

León, J. (1953). Alfaroa manningii, una nueva Juglandácea de Costa Rica, Ceiba, 4, 42-47. 
León, J. (1960). Una especie nueva de Guarea (Meliaceae) con hojas de crecimiento indefinido. Revista de Biología Tropical, 8(2), 147-153. Recuperado de: http://goo.gl/DahvX

León, J. (1966). Central American and West Indian species of Inga (Leguminosae). Annals of the Missouri Botanical Garden, 53(3), 265-359. Recuperado de: http://goo.gl/Kc8rFm

León, A. J. (2006). Semblanza sobre Luis Fournier Origgi. Lankesteriana, 6(2), 29-32. Recuperado de: http://goo.gl/6slRX

Tapia, M. E. (2000). Cultivos andinos subexplotados y su aporte a la alimentación. 2da. edición. Santiago de Chile: Organización de las Naciones Unidas para la Agricultura y la Alimentación (FAO), Oficina
Regional de la FAO para América Latina y el Caribe. Recuperado de: http://goo.gl/h5yWj

Ugalde, G. J., \& Gauld, I. (2002). Meniscomorpha leoni. In I. Gauld, C. Godoy, R. Sithole, \& G. J. Ugalde. 2002. The Ichneumonidae of Costa Rica, 4. Memoirs of the American Entomological Institute, 66, 540-541.

Valerio, R. J. (1942a). Informe rendido a la Secretaría de Educación Pública sobre la labor realizada en 1940. San José, Costa Rica: Museo Nacional. Serie de Historia del Museo, I(III), 4.

Valerio, R. J. (1942b). Informe rendido a la Secretaría de Educación Pública sobre la labor realizada en 1941. San José, Costa Rica: Museo Nacional. Serie de Historia del Museo, I(IV), 3.

Zamora, V. N. (1990, publ. 1991). Nuevas especies de Inga Miller (Mimosaceae) para Mesoamérica. Brenesia, 33, 99-118. Recuperado de: http://goo.gl/aCP6q

Jaime E. García G. Escuela de Biología de la Universidad de Costa Rica, 2060 San José, Costa Rica, biodiversidadcr@gmail.com Centro de Educación Ambiental de la Universidad Estatal a Distancia 